\title{
$\$$ Research Square \\ Exposure-lag Response of Air Temperature on COVID-19 Incidence in Italy
}

\section{Fang Chyi Fong}

Newcastle University Medicine Malaysia

Michaela Louise Goodson

Newcastle University Medicine Malaysia

Daniel Robert Smith ( $\boldsymbol{D}$ daniel.smith6@newcastle.edu.my )

Newcastle University Medicine Malaysia

\section{Research Article}

Keywords: Air temperature, Covid-19 incidence, delayed effects, Italy, time-series, meta-analysis, distributed lag non-linear model

Posted Date: June 2nd, 2021

DOl: https://doi.org/10.21203/rs.3.rs-536878/v1

License: (c) (i) This work is licensed under a Creative Commons Attribution 4.0 International License. Read Full License 
1 Exposure-lag response of air temperature on COVID-19 incidence in Italy

3 Fang Chyi Fong ${ }^{1}$, Michaela Louise Goodson ${ }^{1}$, Daniel Robert Smith ${ }^{1 *}$

4

5

6 Contact details:

7

8 Fang Chyi Fong

9 Email: F.C.Fong2@ newcastle.edu.my

10

11 Michaela Louise Goodson

12 Email: michaela.goodson@ newcastle.edu.my

13

14 Daniel Robert Smith *Corresponding Author

$15 \quad$ ORCID id. 0000000239168041

16 Email: daniel.smith6@newcastle.edu.my

17

18

19

20

Institution:

21

22

${ }^{1}$ Newcastle University Medicine Malaysia,

23 No. 1, Jalan Sarjana 1,

24 Kota Ilmu, EduCity@ Iskandar,

2579200 Iskandar Puteri,

26 Johor,

27 Malaysia.

28

29

30

Keywords

Air temperature, Covid-19 incidence, delayed effects, Italy, time-series, meta-analysis, distributed lag non-linear model 


\section{Exposure-lag response of air temperature on COVID-19 incidence in Italy}

2

3

4

5 The exposure-lag response of air temperature on COVID-19 incidence is unclear and there

6 have been concerns regarding the robustness of previous studies. Here we present an analysis

\section{Abstract} of high spatial and temporal resolution using the distributed lag non-linear modelling (DLNM)

framework. We first fit statistical models to select Italian cities, accounting for lag effects up to 10 days and several categories of potential confounders (policy, mobility, meteorological, and pollution variables). Estimates from these models are then synthesised using random effects meta-analysis to yield pooled estimates of the exposure-lag response presented as the relative risk $(R R)$ and cumulative $R R\left(R_{\text {cum }}\right)$. Though there was variation in the lag-specific exposure-response curves, the cumulative exposure response was approximately bell shaped, with highest risk at $19.8{ }^{\circ} \mathrm{C}, 2.39$ [95\% CI: $\left.1.13 ; 2.94\right]$ times that at $4.7{ }^{\circ} \mathrm{C}$ which represented the lowest risk. Our work is in agreement with studies suggesting "lower" and "higher" temperatures might reduce covid-19 transmission, though our results suggest the optimum temperature for outdoor transmission might be higher than previously thought. Due to this uncertainty, our work underscores the need for facemasks and social distancing even in warm temperatures. 
3 As of May 2021, more than 150 million cases of COVID-19 infections have been confirmed 4 worldwide, and the global death toll has now exceeded 3 million people (World Health 5 Organization, 2020). Aside from the direct impact of COVID-19, healthcare in other fields 6 unrelated to infectious disease have also suffered from its knock-on effects. Woolf et al. (2020) 7 reported a higher number of deaths in the United States than expected when comparing data to 8 previous years, and estimated that $35 \%$ of the excess mortality is due to conditions not directly 9 attributed to COVID-19. Though difficulty in seeking medical treatment due to movement 10 restriction measures and reluctance to risk exposure in healthcare settings are speculated reasons for the excess mortality, an overwhelmed healthcare system likely played a significant role, especially in the formative stages of the outbreak.

Italy was among the worst affected countries near the start of the pandemic, with daily cases peaking at the end of March 2020. Italy remained within the top ten countries with the highest cumulative number of confirmed cases by June 2020, at more than 240,000 cases, with a recorded case fatality rate exceeding $10 \%$ (Worldometer, 2020). Over a year since the start of the pandemic, widespread lockdown was recently reimposed due to transmission rates rising once more (Day 2021)

Since the beginning of the outbreak, a number of studies have investigated the relationship between air temperature and COVID-19 incidence, though the evidence is inconclusive (Dong et al 2021). Studies in Italy are no exception with reports of both positive (Passerini et al., 2020; Zoran et al., 2020) and negative (Pirouz et al., 2020; De Angelis et al 2021) associations. Several studies have also attempted to allow for non-linear and delayed effects of climatic predictors, though until very recently (Nottemyer and Sera 2021) these have been limited to select regions (Runkle et al., 2020), relatively narrow temperature ranges (Passerini et al., 2020; Bashir et al., 2020; Tosepu et al., 2020), relatively short time series (Shi et al., 2020) and lag periods (Tobías and Molina, 2020), all of which may induce significant biases. Indeed, a recent review of papers which have investigated the association between air temperature and COVID-19 incidence identified significant methodological flaws in the majority of papers studied (Dong et al 2021). This is perhaps unsurprising given the urgency for published works. In addition to the limitations highlighted above, concerns highlighted by Dong et al (2021) included: low spatial resolution, failure to account for autocorrelation, failure 
1 to account for various categories of confounding variables (e.g. meteorological, policy, 2 mobility, seasonal trends) and failure to account for lag effects with regards to confounders. and temporal resolution using over a full year's worth of data. We first fit statistical models to select Italian cities, accounting for several categories of potential confounders (policy, mobility, meteorological and pollution variables). Estimates from these models are then synthesised using meta-analysis to yield pooled estimates of the exposure-lag response of air temperature on COVID-19 incidence.

2. Materials and Methods

\section{Data sources}

13 Nine Italian cities were included in this analysis, namely: Bologna, Brescia, Livorno, Milan, 14 Modena, Parma, Prato, Rome and Trieste, selected based on the availability of daily city level data, between 01 Jan 2020 and 24 Apr 2021 inclusive. Daily confirmed COVID-19 cases for each city were obtained using the COVID-19 R package (Guidotti and Ardia, 2020). Daily country-level public policy measures were also obtained from the above source, including: (1) school closure; (2) workplace closure; (3) event cancellations; (4) gathering restrictions; (5) transport closure;

(6) stay-home restrictions;

(7) internal movement restrictions; international movement restrictions; (9) information campaigns; (10) testing policy; (11) contact tracing; and (12) stringency index. Daily median city-level meteorological and pollution data was obtained from https://aqicn.org/data-platform/covid19/ and included: (1) air temperature; (2) carbon monoxide (CO); (3) dew-point temperature; (4) humidity; (5) nitrogen dioxide $\left(\mathrm{NO}_{2}\right)$; (6) ozone $\left(\mathrm{O}_{3}\right)$ (7) PM2.5; (8) PM10, (9) air pressure, (10) sulphur dioxide (SO2), (11) wind-speed; and (12) wind-gust. Daily country-level mobility data were obtained from the google mobility index, including: (1) retail and recreation; (2) grocery and pharmacy; (3) parks, (4) transit stations, (5) workplaces; and (6) residential.

\section{Study design}

We used the two-stage time series design (Berhane and Thomas 2002). In the first stage, a series of city-specific statistical models were fitted, with terms representing the exposure-lag response as well as additional confounding variables. In the second stage, coefficients and covariance matrices from the first stage models were pooled using meta-analysis. This 
1 approach controls for city-level confounders, relaxes the strong assumption that the exposure-

2 lag response is not city-specific and has been deployed in a recent similar study (Nottmeyer and Sera 2021).

First stage analysis

6 Due to the large number of potential confounding variables, we performed a redundancy analysis using $\mathrm{R}^{2}=0.8$ as the cut-off, to remove variables that could be strongly predicted from those remaining (Harrell 2015). In order to model the temporal dependency between daily temperature and COVID-19 incidence, we adopted the Distributed Lag Non-linear Model (DLNM) framework (Gasparrini et al., 2010). For each city, we fitted a Generalized Additive Model (GAM) of the form:

$Y_{t} \sim$ quasiPoisson $\left(\mu_{t}\right)$,

where $Y_{t}$ denotes the COVID-19 cases on day $t$ and $\beta_{0}$ the model intercept. $T_{t}, l$ denotes a crossbasis for temperature on day $t$ considering lag $l$, constructed using natural splines with 3 prespecified degrees of freedom for both the exposure and lag bases (Nottmeyer and Sera 2021 found that larger values for the degrees of freedom incurred a loss of precision for the estimates) with $\boldsymbol{\beta}_{1}$ the corresponding vector of coefficients. We considered lag range 0 through 10 days inclusive as in recent studies (Lauer et al., 2020; Nottmeyer and Sera 2021). dow denotes the day of week fitted as dummy variables, with $\boldsymbol{\beta}_{\mathbf{2}}$ the corresponding vector of coefficients. $u_{t k}$ denotes additional predictors fitted as penalised smoothing splines. Of these smooth terms, time, $t$ was fitted as a cubic regression spline with 9 degrees of freedom to control for seasonal and long term trends, whilst the remaining terms were fitted as penalized cross-basis splines (Gasparrini et al. 2017) in order to account for lag effects in these predictors. Specifically, grocery, work, park, humidity, PM10, pressure, wind-speed and wind-gust were fitted using penalized cubic regression splines with 3 degrees of freedom for both the exposure and lag dimensions (total $=9$ degrees of freedom for each term). Transport closing and internal movement restrictions were fitted using dummy coding for the exposure dimension and natural splines for the lag dimension, with 3 pre-specified degrees of freedom. To avoid overfitting and potential multicollinearity, additional penalties were added to the null space of the bases for all smooth terms, allowing them to shrink to zero (Marra and Wood 2011). However, we 
1 did not shrink the cross-basis for air temperature since this was the predominant term of interest 2 in our model (Harrell 2015). Heteroscedasticity and Autocorrelation Consistent (HAC) 3 estimation of the covariance matrix was performed using the sandwich estimator (Zeileis 2004, 4 2006). Parameters were estimated using quasi- restricted maximum likelihood (REML) 5 (McCullagh and Nelder, 1989). We performed a sensitivity analysis by altering the lag period 6 to 0-5 and 0-15 days respectively as in Nottmeyer and Sera (2021).

10 Second stage analysis

11 For each of the city-specific GAM's, the cross-basis $T_{t}, l$ was reduced to simpler sets of one12 dimensional coefficients and corresponding covariances for the exposure- and lag- dimensions respectively (Gasparrini and Armstrong 2013). These were then pooled using random effects meta-analysis with REML estimation (Sera et al 2019). We computed the Relative Risk (RR) ( $\pm 95 \%$ confidence intervals) and cumulative $\mathrm{RR}\left(\mathrm{RR}_{\text {cum }}\right)$ to quantify the direction and

\section{Results}

Across all city-specific time series', daily median air temperature ranged from -2.2 to $31.2{ }^{\circ} \mathrm{C}$. magnitude of the exposure- and lag- response respectively. For the exposure response, predictions were centred at the air temperature which yielded the minimum $R_{\text {cum }}$ (Nottmeyer and Sera 2021).

All statistical analyses were performed in R version 3.6.1 (R Core Team, 2013), relying heavily on the packages 'dlnm' (Gasparrini, 2011), 'mgcv' (Wood, 2011) and 'mixmeta' (Sera et al., 2019).

The air temperature yielding minimum $\mathrm{RR}_{\text {cum }}$ was $4.7^{\circ} \mathrm{C}$; all exposure response functions were therefore centred at this point.

The exposure response curves varied according to the lag period (fig 1). At early lags, the RR increased from 1 at $4.7^{\circ} \mathrm{C}$ (reference level) to peak at $19.8{ }^{\circ} \mathrm{C}$, before declining at higher temperatures. At lag 6 days, the RR increased linearly from the reference level as temperatures increased. At late lags, the RR largely remained close to 1 with increasing temperatures, though there was a decline below 1 at high temperatures by lag 10 days. The RR remained close to 1 
1 for decreases in temperature compared below the reference level, though there is the possibility

2 of a slight increase by the end of the lag period.

3 The lag-specific effects were summed to yield the cumulative exposure response curve (fig 2).

4 For temperatures above the reference level, the cumulative exposure response function

5 resembled a bell-shaped curve; with $\mathrm{RR}_{\text {cum }}$ increasing to peak at $19.8{ }^{\circ} \mathrm{C}$ before declining to

$6 \quad \mathrm{RR}_{\text {cum }} \sim 1$ at $30{ }^{\circ} \mathrm{C}$. For temperatures below the reference level, $\mathrm{RR}_{\text {cum }}$ increased slightly.

7 The exposure-specific lag-response curves are shown in fig 3. Small increments either below

8 or above the reference temperature produced little effect and the RR's were constant across the

9 lag period. For larger increases in temperature, the RR increased to some peak before declining

10 at the end of the lag period, with the suggestion that the peak occurs later for larger increments

11 in temperature compared to the reference level. Examining the cumulative effects plot (fig 4),

12 the $\mathrm{RR}_{\text {cum }}$ appears to monotonically increase over the lag period for 14 and $21^{\circ} \mathrm{C}$, though there

13 is the suggestion of a plateauing effect in the latter. For air temperature $=28{ }^{\circ} \mathrm{C}$, the $\mathrm{RR}_{\text {cum }}$

14 peaks at around lag=8 days before declining to lag=10 days.

15 Shortening the modelled lag period from 0-10 to 0-5 days resulted in a sigmoidal shaped 16 cumulative exposure response, with air temperature corresponding to maximal risk somewhat 17 higher (approx. $25^{\circ} \mathrm{C}$ ) (Supplementary information fig 1). However, the confidence intervals 18 do not rule out a declining $\mathrm{RR}_{\text {cum }}$ at higher temperatures (as in the main analysis) and there 19 appeared to be increasing uncertainty at high temperatures with widening confidence intervals. 20 However, the maximal $\mathrm{RR}_{\text {cum }}$ is similar in magnitude to the main analysis. Lengthening the modelled lag period from 0-10 to 0-15 days did not change the shape of the cumulative exposure response curve (Supplementary information fig 2). However, it appeared somewhat flatter, with a slightly lower maximal $R_{\mathrm{cum}}=\sim 2$. However, this maximal risk occurred at approximately the air temperature as in the main analysis. There also appeared to be a loss of precision incurred by extending the lag period, with wider confidence intervals than in the corresponding main analysis. 
$\operatorname{Lag}=2$ days

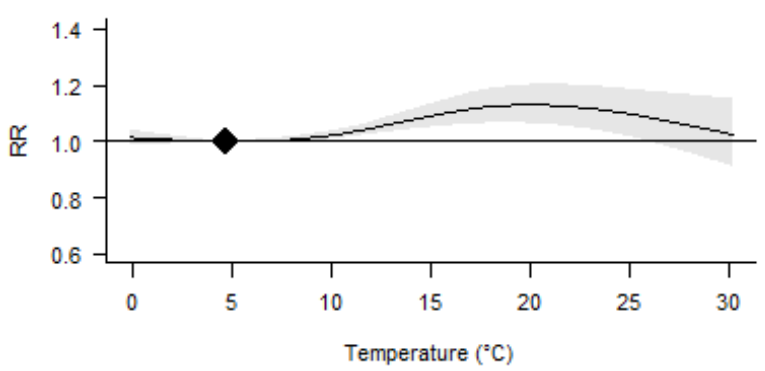

$\operatorname{Lag}=6$ days

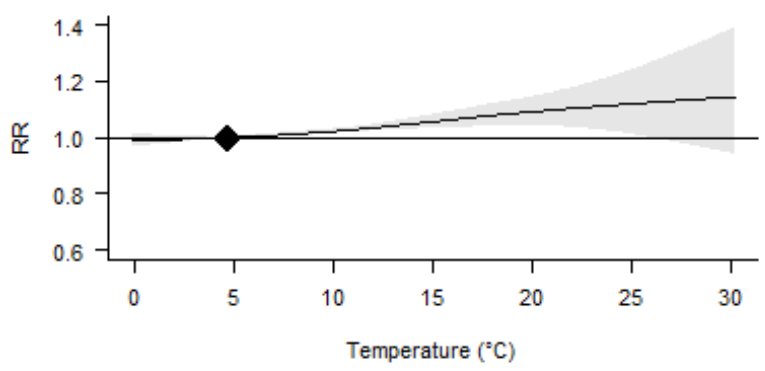

$\operatorname{Lag}=10$ days

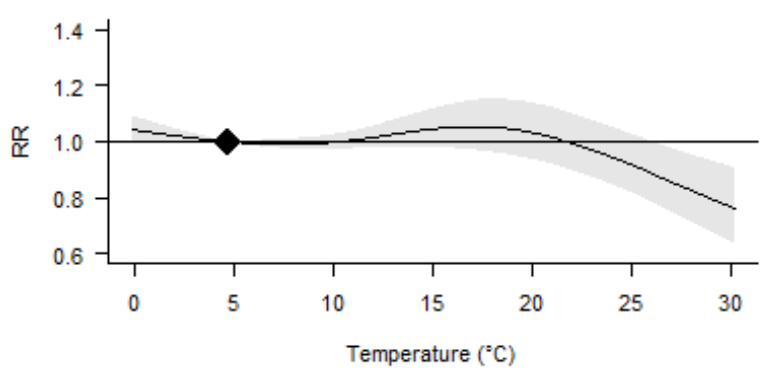

$\operatorname{Lag}=4$ days

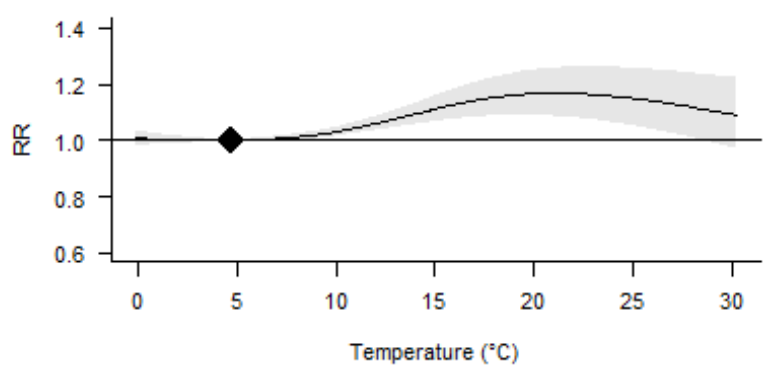

Lag $=8$ days

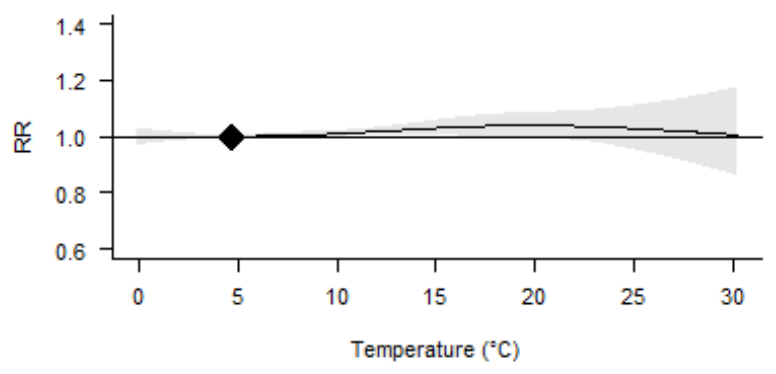

3 Figure 1. Lag-specific effects of the exposure-response association. The effects are centred at 4 the reference level ( 5 intervals. 


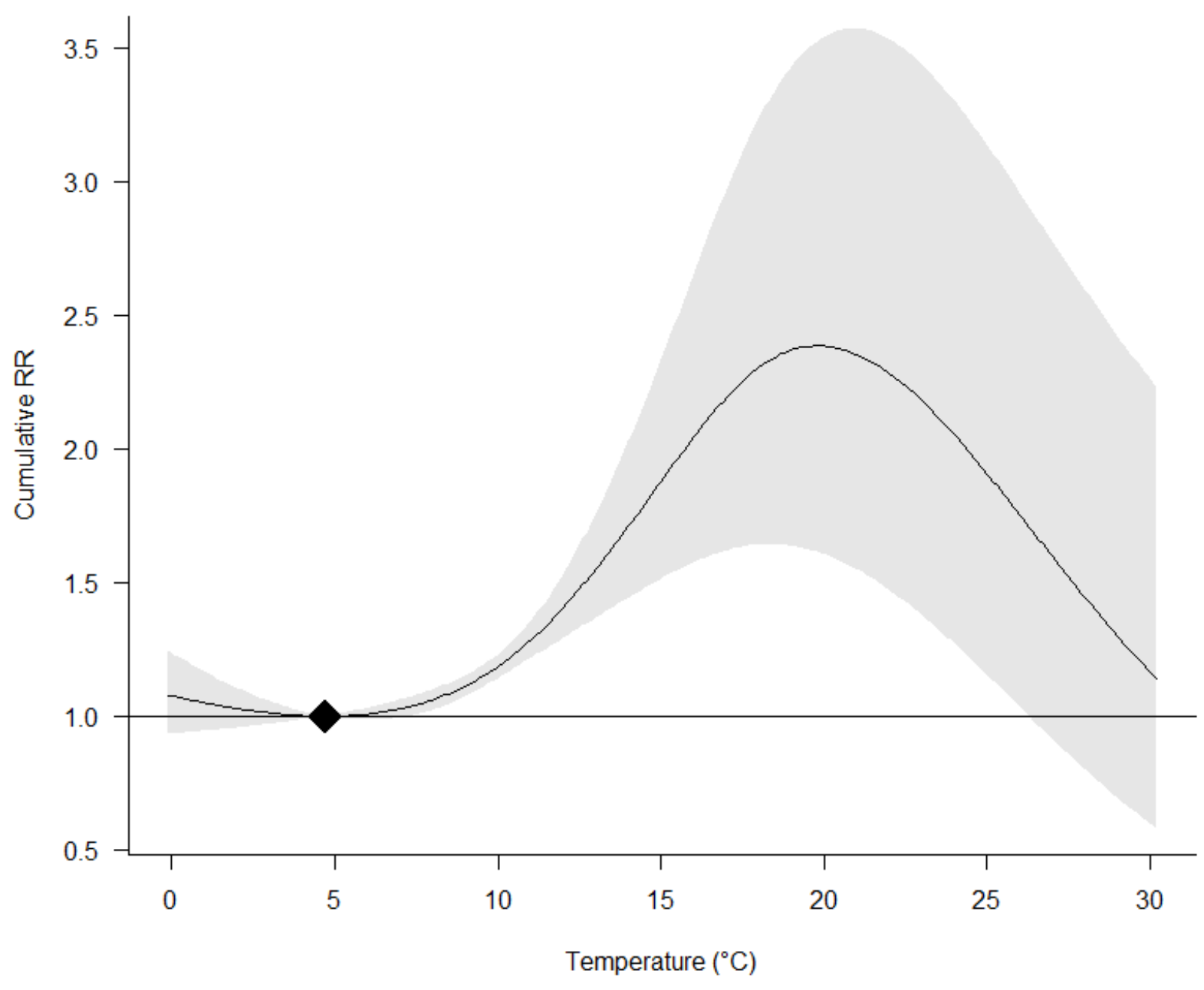

2 Figure 2. Overall cumulative exposure-response association. Cumulative RR is computed by 3 summing the log RR over the lag period ( 0 through 10 days inclusive). The effects are centred 4 at the reference level (solid diamond $=4.7^{\circ} \mathrm{C}$ ). Grey shaded area represents $95 \%$ confidence 5 intervals.

6

7

8 
Temperature $=0{ }^{\circ} \mathrm{C}$

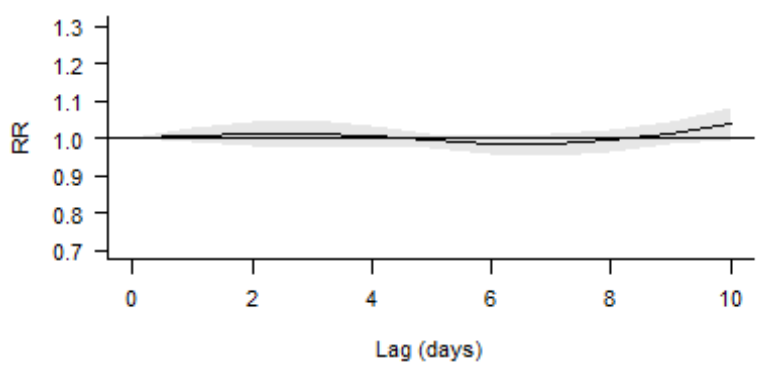

Temperature $=14^{\circ} \mathrm{C}$

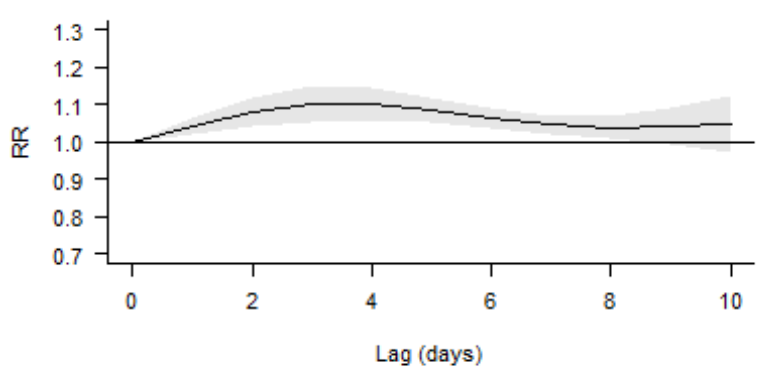

Temperature $=28^{\circ} \mathrm{C}$

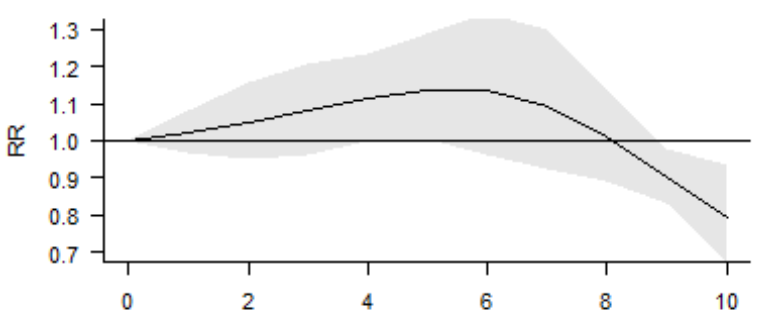

Lag (days)
Temperature $=7^{\circ} \mathrm{C}$

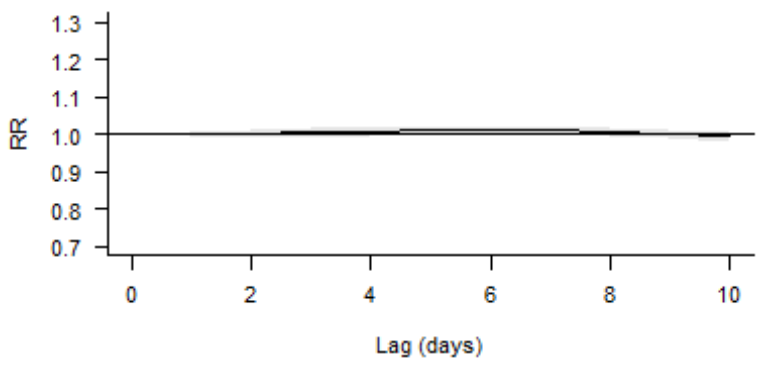

Temperature $=21^{\circ} \mathrm{C}$

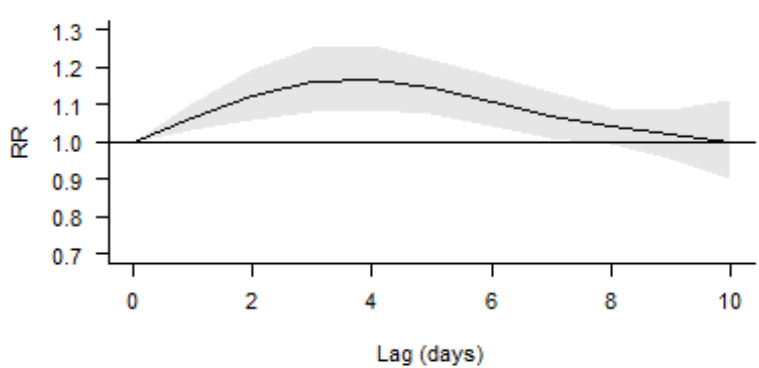

1

2 Figure 3. Exposure-specific effects of the lag-response association. The effects are centred at 3 lag $=0$ and temperature $=4.7^{\circ} \mathrm{C}$. Grey shaded area represents $95 \%$ confidence intervals. 

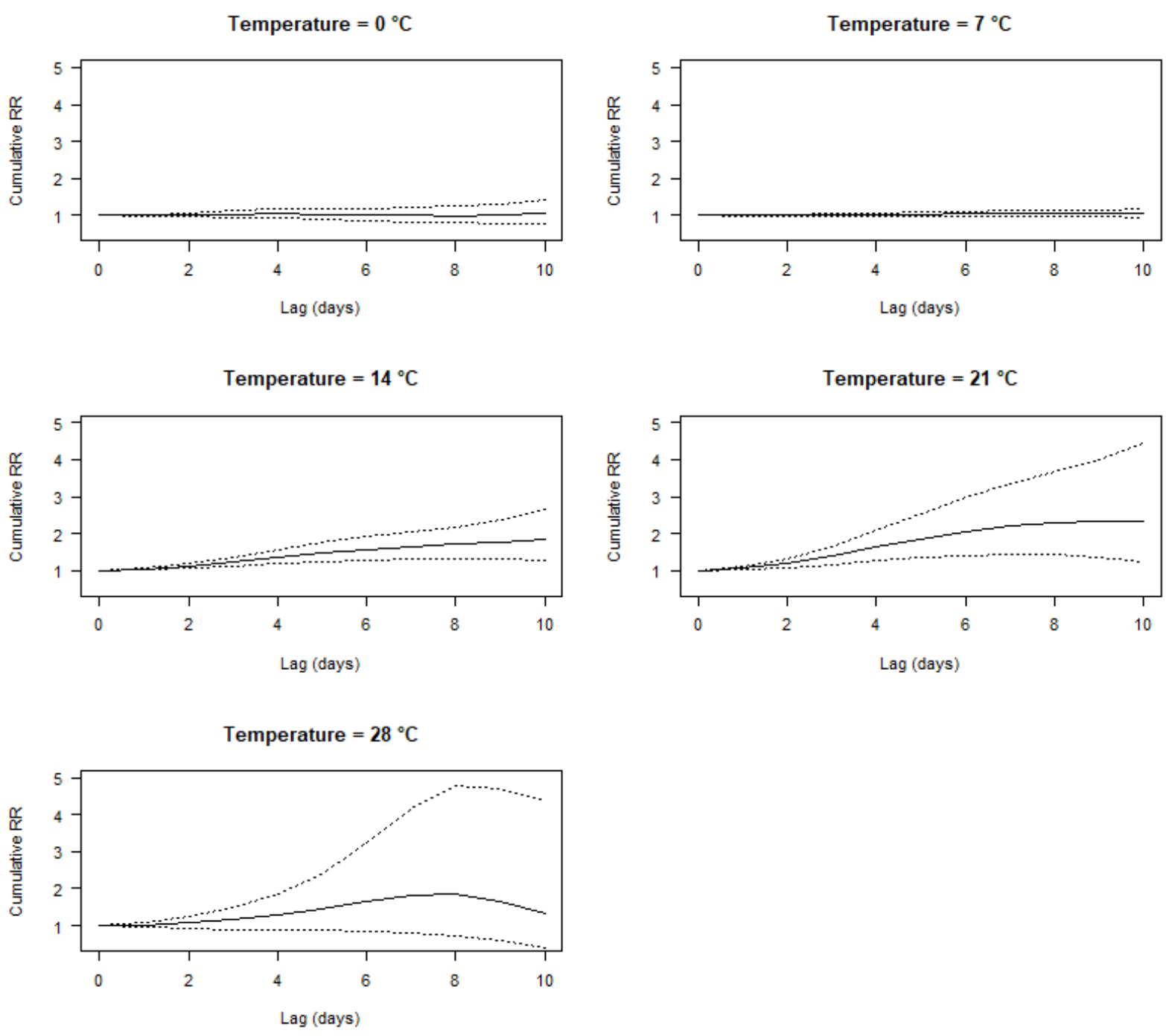

2 Figure 4. Overall exposure-specific lag-response association. The effects are centred at lag=0 3 days and temperature $=4.7^{\circ} \mathrm{C}$. Cumulative RR is computed by summing the log RR over the 4 lag period (0 through 10 days inclusive). Dashed lines represent 95\% confidence intervals. 
2 In this contribution, we quantified the exposure-lag response of air temperature on COVID-19

3 incidence in Italy. We fitted a series of city-specific statistical models to extended time series

4 accounting for numerous confounders, before pooling the effects using meta-analysis. We

5 found a non-linear exposure-lag response with minimal and maximal risk at 4.9 and $19.8{ }^{\circ} \mathrm{C}$

6 respectively.

7 Our study supports a growing body of evidence of a non-linear association between 8 temperature and COVID-19 incidence (Runkle et al 2020; Shi et al 2020; Gao et al 2021;

9 Nottmyer and Sera 2021; Yuan et al 2021). Nottmyer and Sera (2021) performed a similar

10 study on cities in England. The shape of the non-linear exposure-response relationship reported 11 herein is in agreement this study suggesting "lower" and "higher" temperatures might reduce 12 COVID-19 transmission. However, it appeared as though the peak risk was approximately 6 $13{ }^{\circ} \mathrm{C}$ higher in our study $\left(19.8^{\circ} \mathrm{C}\right)$ compared to theirs $\left(11.9{ }^{\circ} \mathrm{C}\right)$. Moreover, the maximal $\mathrm{RR}_{\text {cum }}$ 14 reported herein $(2.39$ [95\% CI: $1.13 ; 2.94])$ is also somewhat larger than that of the above study 151.62 [95\% CI: 1.44; 1.81). The maximum RR for our exposure-specific lag response curves 16 falls between approximately 3 and $6{ }^{\circ} \mathrm{C}$ and is consistent with Nottmyer and Sera (2021).

Experiments on SARS-CoV-2 have demonstrated a temperature-dependent effect on the stability of virus particles on smooth surfaces (Chin et al., 2020). Since SARS-CoV-2 is shown to be capable of surviving in aerosols for at least 3 hours, airborne transmission is highly plausible (van Doremalen et al., 2020). Aerosol dynamics, and subsequently disease transmission, appear to be affected by air temperature and humidity. Low humidity and low temperature result in smaller infected droplet nuclei that can travel further, remain airborne for a longer duration, and cause delayed virus inactivation (Chen, 2020; Moriyama et al., 2020). Experimental evidence for the effects of climate on transmission dynamics using influenza virus in a guinea pig model lends credence to this hypothesis (Lowen et al., 2007). Low temperature and high humidity favour contact transmission (Zhao et al., 2020; Lowen et al., 2007), whilst high temperature and low humidity promote the accumulation of aerosol particles, increasing the likelihood of inhalation (Nottemeyer and Sera 2021). As highlighted in the above reference, this ambiguity makes non-linear associations plausible. However, not all experimental studies agree that stability of SARS-CoV-2 is greatly affected by temperature, so caution is warranted in drawing definite conclusions (Kratzel et al., 2020). 
1 Our study has several strengths which we believe make it a valuable contribution to the 2 literature, particularly in the light of criticism raised by Dong et al (2021). First, we employ the 3 DLNM framework to simultaneously assess non-linear and delayed dependencies in the 4 exposure and lag dimensions (Gasparini et al 2010). Second, we used city-level daily data for 5 COVID-19 incidence and a number of confounders. Third, we used the powerful two-stage 6 design; this allows for both the background risk and the exposure lag response functions to 7 vary by city which would otherwise incur ecologic bias. Forth, we account for a large number of confounders including meteorological, pollution, policy and mobility and allow for delayed dependencies in these effects. Fifth, the precision of our estimates are robust to heteroskedasticity and autocorrelation. Sixth, we controlled for overfitting using shrinkage estimators. In sum, we have addressed all the main concerns highlighted in a recent review of the relationship between air temperature and COVID-19 incidence (Dong et al 2021).

13 However, our work is not without limitations. The first concerns the accuracy of the estimated daily counts of COVID-19. Notwithstanding the rate of testing, it is postulated that estimates are biased low due to undocumented infections (Li et al., 2020) or other reporting errors (De Natale et al., 2020). Secondly, our study is limited to the lag effects of median daily temperature. A recent study (Babin, 2020) reported that extremes of daily temperature might actually be a better predictor of COVID-19 incidence; this would be a worthy avenue for future research. Third, our analysis was based on a smaller number of cities than similar work (Shi et 2020; Nottemyer and Sera 2021); this was a direct consequence of the availability of city level data for confounders which we considered a key feature of our analysis. Although metaanalysing 9 cities in this framework is valid (the example in Gasparini and Armstrong 2013 used 10 regions), further work might consider a larger sample size of cities to yield more precise effect estimates. Forth, although meteorological and pollution data were city level, policy and mobility data were country level which may incur some aggregation bias.

In conclusion, our results support previous work of a non-linear exposure-lag response of air temperature on COVID-19 incidence, with "low" and "high" temperatures less favourable to outdoor transmission. However, our finding of maximal risk at $19.8{ }^{\circ} \mathrm{C}$ is somewhat higher than similar recent work. If our findings are correct, this implies that only at

30 higher temperatures $\left(>20^{\circ} \mathrm{C}\right)$ might the risk of COVID-119 infection start to reduce. Due to 31 this uncertainty, this work underscores the need for facemasks and social distancing even in warm temperatures. 


\section{Conflicts of interest}

2 None

\section{Author Contributions Statement}

7 D.R.S, F.C.F and M.L.G designed the study. F.C.F and D.R.S wrote the main manuscript text.

8 D.R.S conducted the statistical analysis and prepared the figures. All authors were involved in 9 reviewing the final manuscript.

\section{Competing interests}

11 We declare no competing interests as defined by Nature Research, or other interests that might 12 be perceived to influence the results and/or discussion reported in this paper.

\section{References}

Babin, S., 2020. 'Use of Weather Variables in SARS-CoV-2 Transmission Studies', Int J Infect Dis, 100, pp. 333-336. https://doi.org/10.1016/j.ijid.2020.09.032.

Balmer, C, Pollina, E., 2020. 'Italy threatens to ban outdoor exercise as coronavirus deaths surge', Reuters, 2020 Mar 18. Available at: https://www.reuters.com/article/us-healthcoronavirus-italy-idUSKBN2151M8.

Bashir, M. F., Ma, B., Bilal, et al., 2020. 'Correlation between climate indicators and COVID-19 pandemic in New York, USA', Sci Total Environ, 728, pp. 138835. https://doi.org/10.1016/j.scitotenv.2020.138835.

Berhane K, Thomas DC (2002) A two-stage model for multiple time series data of counts. Biostatistics. 3(1):21-32. doi: 10.1093/biostatistics/3.1.21.

Brooks, M. E., Kristensen, K., van Benthem, K. J., et al., 2017. 'glmmTMB balances speed and flexibility among packages for zero-inflated generalized linear mixed modeling', The 
Bruns, D. P., Kraguljac, N. V., Bruns, T. R., 2020. 'COVID-19: Facts, Cultural

2 Considerations, and Risk of Stigmatization', J Transcult Nurs, 31(4), pp. 326-332.

3 https://doi.org/10.1177/1043659620917724.

Casanova, L. M., Jeon, S., Rutala, W. A., et al., 2010. 'Effects of air temperature and relative humidity on coronavirus survival on surfaces', Appl Environ Microbiol, 76(9), pp. 2712-7. https://doi.org/10.1128/AEM.02291-09.

Chan, K. H., Peiris, J. S., Lam, S. Y., et al., 2011. 'The effects of temperature and relative humidity on the viability of the SARS coronavirus', Adv Virol, 2011, pp. 734690. https://doi.org/10.1155/2011/734690.

Chen, L. D., 2020. 'Effects of ambient temperature and humidity on droplet lifetime A perspective of exhalation sneeze droplets with COVID-19 virus transmission', Int J Hyg Environ Health, 229, pp. 113568. https://doi.org/10.1016/j.ijheh.2020.113568.

Cherrie, M. P. C., Nichols, G., Iacono, G. L., et al., 2018. 'Pathogen seasonality and links with weather in England and Wales: a big data time series analysis', BMC Public Health, 18(1), pp. 1067. https://doi.org/10.1186/s12889-018-5931-6.

Chin, A. W. H., Chu, J. T. S., Perera, M. R. A., et al., 2020. 'Stability of SARS-CoV-2 in different environmental conditions', Lancet Microbe, 1(1), pp. e10. https://doi.org/10.1016/S2666-5247(20)30003-3.

Day, M. (2021). Covid-19: Italy reimposes widespread lockdown as transmission rate rises again. BMJ; 372:n726 doi: https://doi.org/10.1136/bmj.n726

De Natale, G., Ricciardi, V., De Luca, G., et al., 2020. 'The COVID-19 Infection in Italy: A Statistical Study of an Abnormally Severe Disease', J Clin Med, 9(5). https://doi.org/10.3390/jcm9051564.

Denti, A., Amante, A., 2020. 'Lockdown: a day in the life of a teacher in Italy's coronavirus red zone', Reuters, 2020 Feb 25. Available at: https://uk.reuters.com/article/uschina-health-italy-red-zone-diary-idUKKCN20I2A5.

Dong Z, Fan X, Wang J, Mao Y, Luo Y, Tang S (2021) Data-related and 28 methodological obstacles to determining between temperature and COVID-19 transmission. Environ. Res. Lett. 16 (2021) 034016. 
Gao M, Zhou Q, Yang X,et al. Nonlinear modulation of COVID-19transmission by 2 climate conditions. Meteorol Appl.2021;28:e1985.https://doi.org/10.1002/met.19856of6 GAO

3 ET AL.Meteorological ApplicationsScience and Technology for Weather and Climate

4

Gasparrini, A., 2011. 'Distributed Lag Linear and Non-Linear Models in R: The Package dlnm', J Stat Softw, 43(8), pp. 1-20.

Gasparrini, A., Armstrong, B., Kenward, M. G., 2010. 'Distributed lag non-linear models', Stat Med, 29(21), pp. 2224-34. https://doi.org/10.1002/sim.3940.

Gasparrini, A., Armstrong, B. Reducing and meta-analysing estimates from distributed lag non-linear models. 2013. BMC Med Res Methodol 13, 1. https://doi.org/10.1186/14712288-13-1.

Gasparrini A., Scheipl F, Armstrong B, Kenward MG. 2017. A penalized framework for distributed lag non-linear models, Biometrics 73(3), 938-948.

Grassly, N. C., Fraser, C., 2006. 'Seasonal infectious disease epidemiology', Proc Biol Sci, 273(1600), pp. 2541-50. https://doi.org/10.1098/rspb.2006.3604.

Guidotti, E., Ardia, D., 2020. 'COVID-19 Data Hub', Journal of Open Source Software, 5(51), pp. 2376. https://doi.org/10.21105/joss.02376.

Harrell, F., 2015. Regression Modeling Strategies: With Applications to Linear Models, Logistic and Ordinal Regression, and Survival Analysis. 2nd edn. Basel, Switzerland: Springer International Publishing AG.

Hartig, F., 2020. DHARMa: residual diagnostics for hierarchical (multi-level/mixed) regression models. Available at: https://cran.rproject.org/web/packages/DHARMa/vignettes/DHARMa.html.

Islam, N., Shabnam, S., Erzurumluoglu, A. M., 2020. 'Temperature, humidity, and wind speed are associated with lower Covid-19 incidence', medRxiv. https://doi.org/10.1101/2020.03.27.20045658.

Kasem, S., Qasim, I., Al-Doweriej, A., et al., 2018. "The prevalence of Middle East respiratory Syndrome coronavirus (MERS-CoV) infection in livestock and temporal relation to locations and seasons', J Infect Public Health, 11(6), pp. 884-888. https://doi.org/10.1016/j.jiph.2018.01.004. 
1 Killerby, M. E., Biggs, H. M., Haynes, A., et al., 2018. 'Human coronavirus circulation 2 in the United States 2014-2017', J Clin Virol, 101, pp. 52-56. 3 https://doi.org/10.1016/j.jcv.2018.01.019.

Kratzel, A., Steiner, S., Todt, D., et al., 2020. 'Temperature-dependent surface stability of SARS-CoV-2', J Infect, 81(3), pp. 452-482. https://doi.org/10.1016/j.jinf.2020.05.074.

Kristensen, K., 2020. Covariance structures with glmmTMB. Available at: https://cran.r-project.org/web/packages/glmmTMB/vignettes/covstruct.html.

Lauer, S.A., et al., 2020. The incubation period of coronavirus disease 2019 (CoVID-

9 19) from publicly reported confirmed cases: estimation and application. Ann. Intern. Med. 172, $10 \quad 577-582$.

Li, R., Pei, S., Chen, B., et al., 2020. 'Substantial undocumented infection facilitates the rapid dissemination of novel coronavirus (SARS-CoV-2)', Science, 368(6490), pp. 489-493. https://doi.org/10.1126/science.abb3221.

Liu, J., Zhou, J., Yao, J., et al., 2020. 'Impact of meteorological factors on the COVID19 transmission: A multi-city study in China', Sci Total Environ, 726, pp. 138513. https://doi.org/10.1016/j.scitotenv.2020.138513.

Lowen, A. C., Mubareka, S., Steel, J., et al., 2007. 'Influenza virus transmission is dependent on relative humidity and temperature', PLoS Pathog, 3(10), pp. 1470-6. https://doi.org/10.1371/journal.ppat.0030151.

Luo, W., Majumder, M. S., Liu, D., et al., 2020. 'The role of absolute humidity on transmission rates of the COVID-19 outbreak', medRxiv. https://doi.org/10.1101/2020.02.12.20022467.

Marra G and Wood SN. 2011 Practical variable selection for generalized additive models. Computational Statistics \& Data Analysis, vol. 55, issue 7, 2372-2387.

McAloon, C., Collins, Á., Hunt, K., et al., 2020. 'Incubation period of COVID-19: a rapid systematic review and meta-analysis of observational research', BMJ Open, 10(8), pp. e039652. https://doi.org/10.1136/bmjopen-2020-039652. 
Moriyama, M., Hugentobler, W. J., Iwasaki, A., 2020. 'Seasonality of Respiratory Viral

2 Infections', Annu Rev Virol, 7(1), pp. 83-101. https://doi.org/10.1146/annurev-virology-

$3 \quad \underline{012420-022445}$.

[dataset] NOAA-ESRL Physical Sciences Laboratory (NOAA-PSL). CPC Global Daily Temperature. Boulder, Colorado, USA: NOAA-ESRL Physical Sciences Laboratory. Available at: https://psl.noaa.gov/data/gridded/data.cpc.globaltemp.html.

Nottmeyer LN \& Sera F 2021. Influence of temperature, and of relative and absolute humidity on COVID-19 incidence in England - A multi-city time-series study. Environmental Research 196,110977.

Passerini, G., Mancinelli, E., Morichetti, M., et al., 2020. 'A Preliminary Investigation on the Statistical Correlations between SARS-CoV-2 Spread and Local Meteorology', Int J Environ Res Public Health, 17(11), pp. 4051. https://doi.org/10.3390/ijerph1711405.

Pirouz, B., Shaffiee Haghshenas, S., Piro, P., 2020. 'Development of an Assessment Method for Investigating the Impact of Climate and Urban Parameters in Confirmed Cases of COVID-19: A New Challenge in Sustainable Development', Int J Environ Res Public Health, 17(8), pp. 2801. https://doi.org/10.3390/ijerph1708280.

Prem, K., Liu, Y., Russell, T. W., et al., 2020. 'The effect of control strategies to reduce social mixing on outcomes of the COVID-19 epidemic in Wuhan, China: a modelling study', Lancet Public Health, 5(5), pp. e261-e270. https://doi.org/10.1016/S2468-2667(20)30073-6.

R Core Team, 2013. R: A language and environment for statistical computing (Version R version 3.6.0 ed). Available at: https://www.r-project.org/.

Runkle, J. D., Sugg, M. M., Leeper, R. D., et al, 2020. 'Short-term effects of specific humidity and temperature on COVID-19 morbidity in select US cities', Sci Total Environ, 740, pp. 140093. https://doi.org/10.1016/j.scitotenv.2020.140093.

Sera, F., Armstrong, B., Blangiardo, M., Gasparrini, A., 2019. An extended mixedeffects framework for meta-analysis. Stat. Med. 38, 5429-5444.

Shi, P., Dong, Y., Yan, H., et al., 2020 a'Impact of temperature on the dynamics of the COVID-19 outbreak in China', Sci Total Environ, 728, pp. 138890. https://doi.org/10.1016/j.scitotenv.2020.138890. 
Shi, P., et al., 2020b. The impact of temperature and absolute humidity on the 2 coronavirus disease 2019 (COVID-19) outbreak - evidence from China. medRxiv. 3 https://doi.org/10.1101/2020.03.22.20038919, 03.22.20038919 (2020).

Tobías, A., Molina, T., 2020. 'Is temperature reducing the transmission of COVID-19?', 5 Environ Res, 186, pp. 109553. https://doi.org/10.1016/j.envres.2020.109553.

Tosepu, R., Gunawan, J., Effendy, D. S., Ahmad, O. A. I., Lestari, H., Bahar, H. and Asfian, P. (2020) 'Correlation between weather and Covid-19 pandemic in Jakarta, Indonesia', Sci Total Environ, 725, pp. 138436. https://doi.org/10.1016/j.scitotenv.2020.138436.

van Doremalen, N., Bushmaker, T., Morris, D. H., et al., 2020. 'Aerosol and Surface Stability of SARS-CoV-2 as Compared with SARS-CoV-1', N Engl J Med, 382(16), pp. 15641567. https://doi.org/10.1056/NEJMc2004973.

van Rij, J., Wieling, M., Baayen, R. H., 2020. itsadug: Interpreting Time Series and Autocorrelated Data Using GAMMs (Version 2.4). Available at: https://CRAN.Rproject.org/package=itsadug.

Vinceti, M., Filippini, T., Rothman, K. J., et al., 2020. 'Lockdown timing and efficacy in controlling COVID-19 using mobile phone tracking', EClinicalMedicine, 25, pp. 100457. https://doi.org/10.1016/j.eclinm.2020.100457. the transmission of COVID-19', SSRN.

Wang, M., Jiang, A., Gong, L., et al., 2020b. 'Temperature significant change COVID19 transmission in 429 cities', medRxiv. https://doi.org/10.1101/2020.02.22.20025791.

Wood, S.N. (2011) Fast stable restricted maximum likelihood and marginal likelihood estimation of semiparametric generalized linear models. Journal of the Royal Statistical Society (B) $73(1): 3-36$

Woolf, S. H., Chapman, D. A., Sabo, R. T., et al., 2020. 'Excess Deaths From COVID19 and Other Causes, March-April 2020', JAMA, 324(5), pp. 510-513. 27 https://doi.org/10.1001/jama.2020.11787.

World Health Organisation, 2020. Coronavirus disease (COVID-19): situation report, 162. World Health Organization. Available at: https://apps.who.int/iris/handle/10665/332970. 
1 Worldometer, 2020. Italy Coronavirus. Available at:

2 https://www.worldometers.info/coronavirus/country/italy/ (Accessed: 2020 July 4 2020).

3 Yuan, S., Jiang, S. C., Li, Z. L., 2020. 'Do Humidity and Temperature Impact the Spread

4 of the Novel Coronavirus?', Front Public Health, 8, pp. 240.

5 https://doi.org/10.3389/fpubh.2020.00240.

Yuan, J., Wu, Y., Jing, W., Liu, J., Du, M., Wang, Y., \& Liu, M. (2021). Non-linear correlation between daily new cases of COVID-19 and meteorological factors in 127 countries. Environmental research, 193, 110521. https://doi.org/10.1016/j.envres.2020.110521

Zeileis, A. 2004. Econometric Computing with HC and HAC Covariance Matrix 10 Estimators. 11, 17, doi:10.18637/jss.v011.i10.

Zeileis, A. 2006. Object-oriented Computation of Sandwich Estimators. 16, 16, 12 doi:10.18637/jss.v016.i09. 14 between surface levels of PM2.5 and PM10 particulate matter impact on COVID-19 in Milan, 15 Italy', Sci Total Environ, 738, pp. 139825. https://doi.org/10.1016/j.scitotenv.2020.139825. 
Figures

$$
\text { Lag }=2 \text { days }
$$

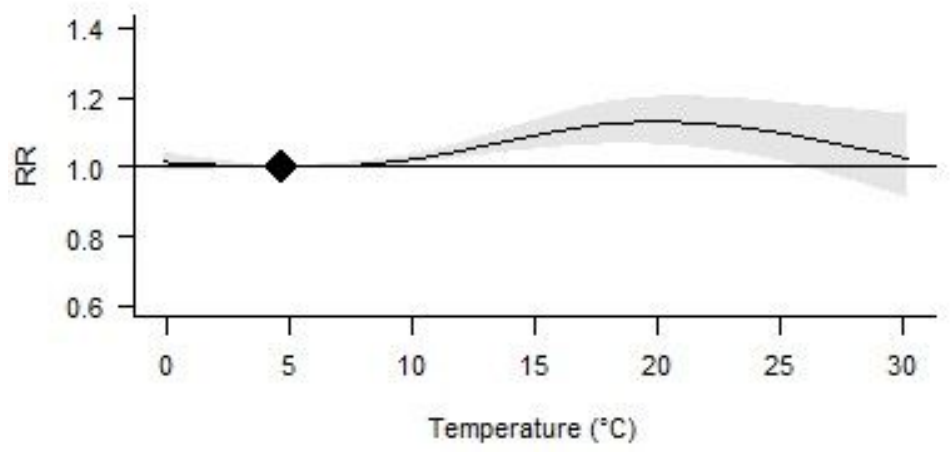

$$
\operatorname{Lag}=6 \text { days }
$$

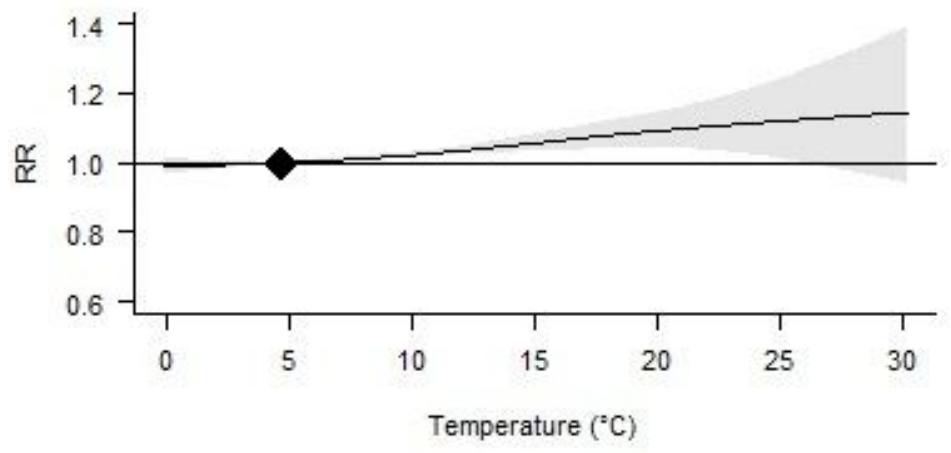

$$
\operatorname{Lag}=10 \text { days }
$$

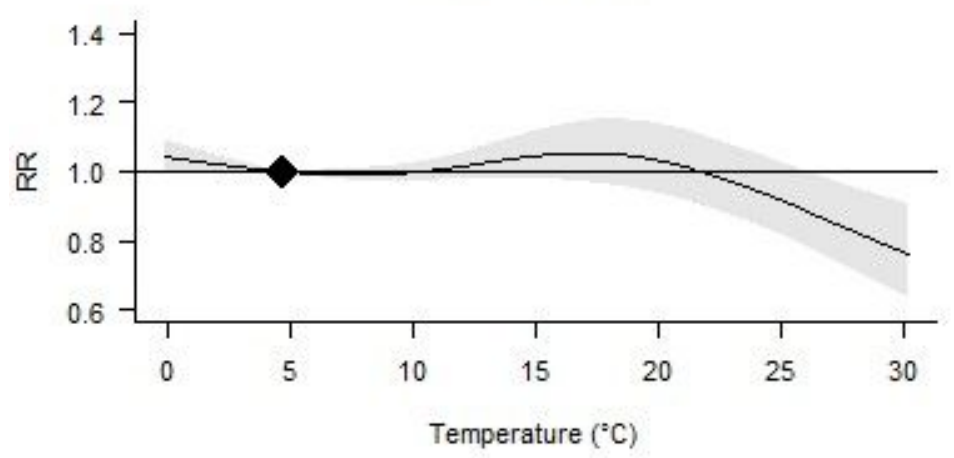

$$
\operatorname{Lag}=4 \text { days }
$$

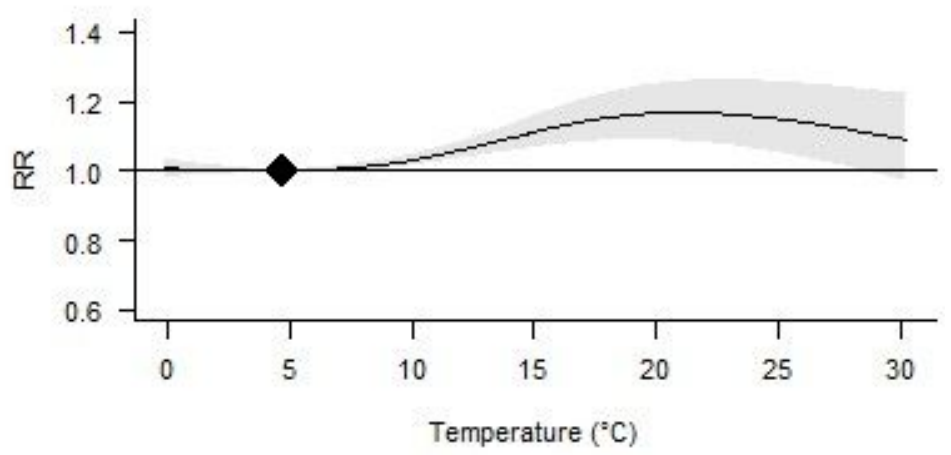

$$
\operatorname{Lag}=8 \text { days }
$$

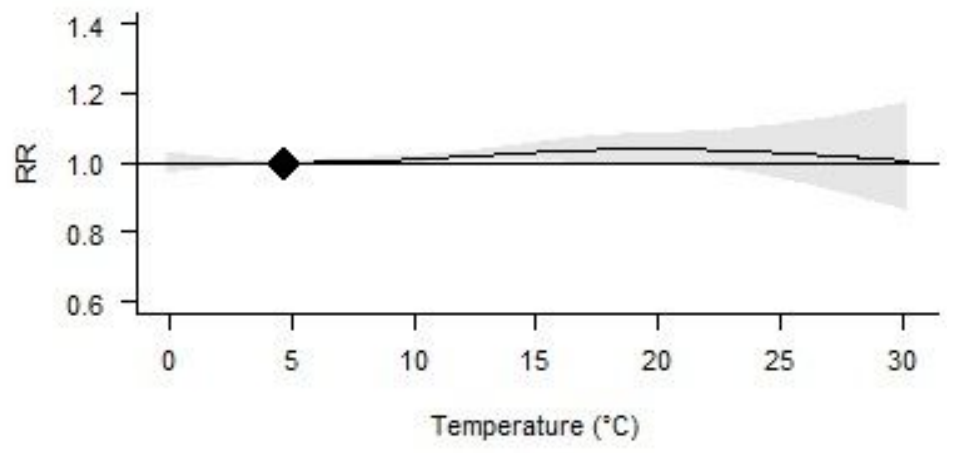

\section{Figure 1}

Lag-specific effects of the exposure-response association. The effects are centred the reference level (solid diamond $=4.7^{\circ} \mathrm{C}$ ). Grey shaded area represents $95 \%$ confidence intervals. 


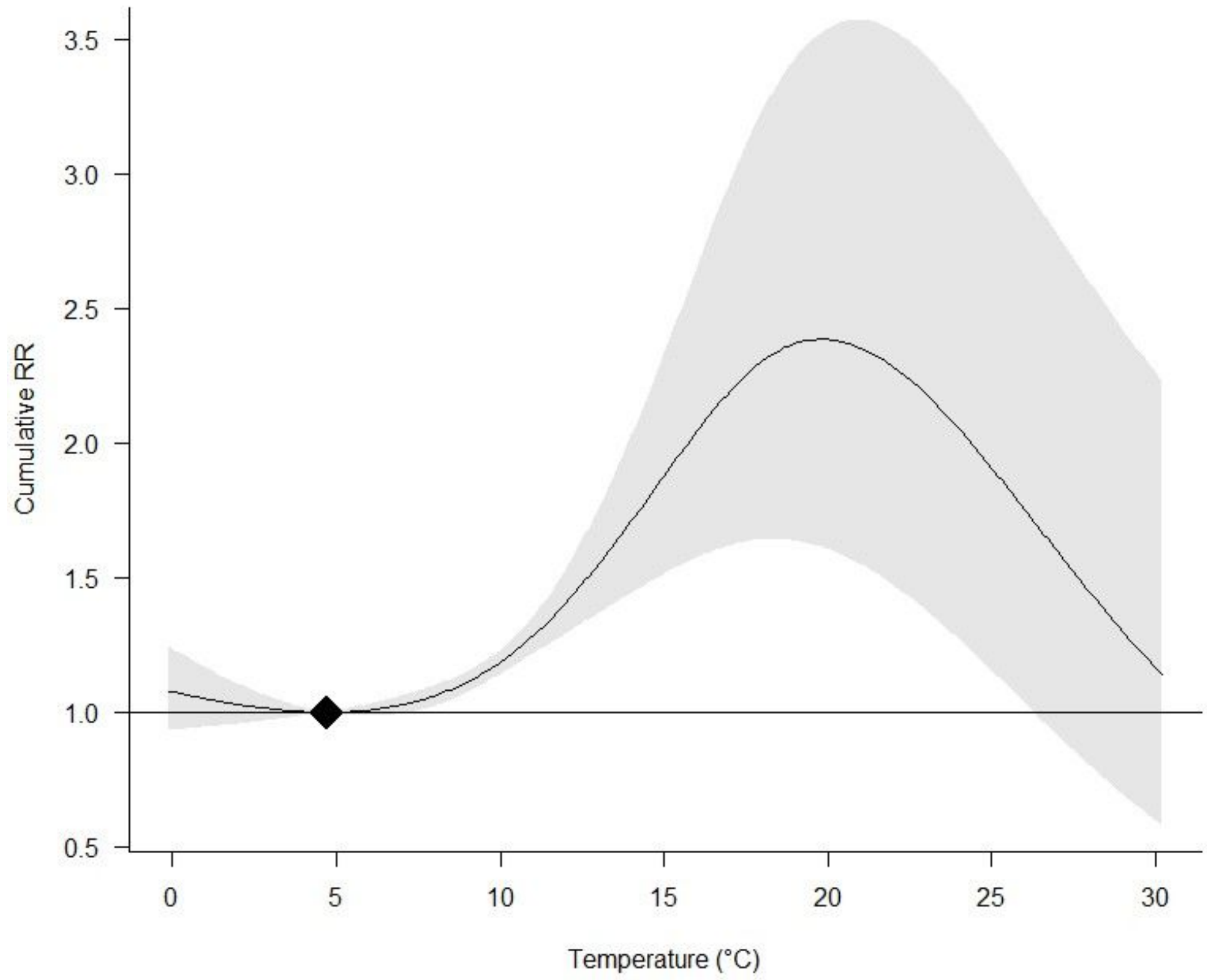

Figure 2

Overall cumulative exposure-response association. Cumulative RR is computed by summing the log RR over the lag period ( 0 through 10 days inclusive). The effects are centred at the reference level (solid diamond $=4.7^{\circ} \mathrm{C}$ ). Grey shaded area represents $95 \%$ confidence intervals. 
Temperature $=0{ }^{\circ} \mathrm{C}$

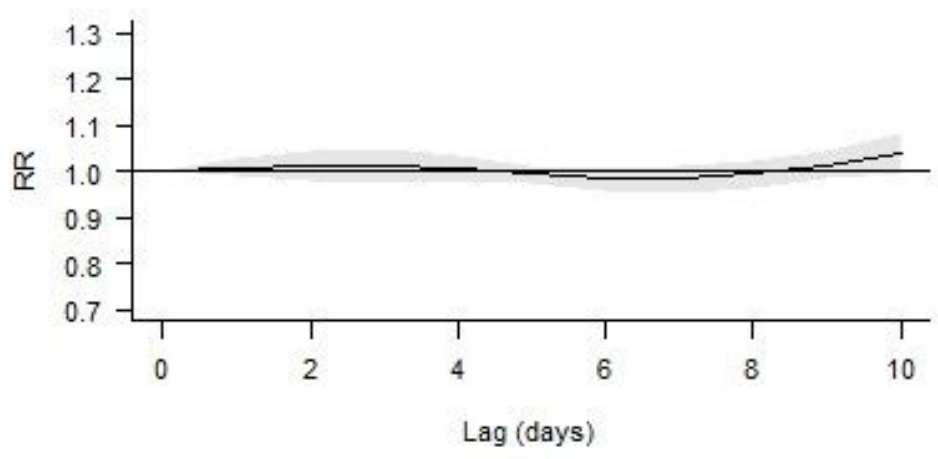

Temperature $=14^{\circ} \mathrm{C}$

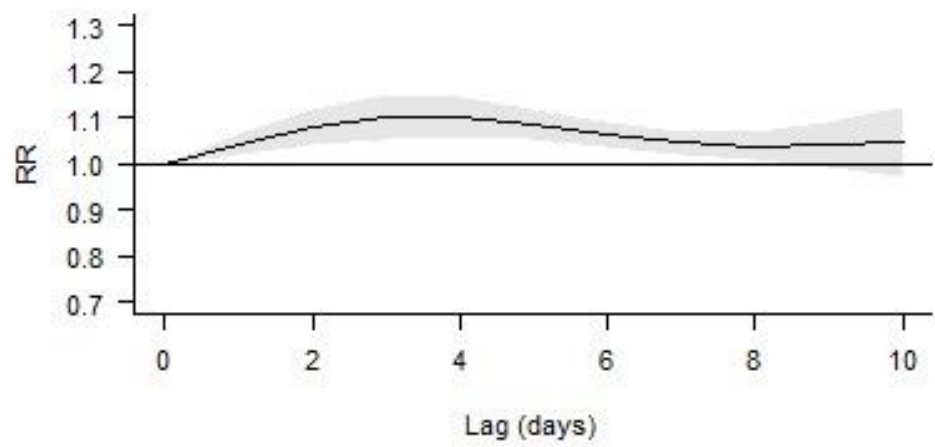

Temperature $=28^{\circ} \mathrm{C}$

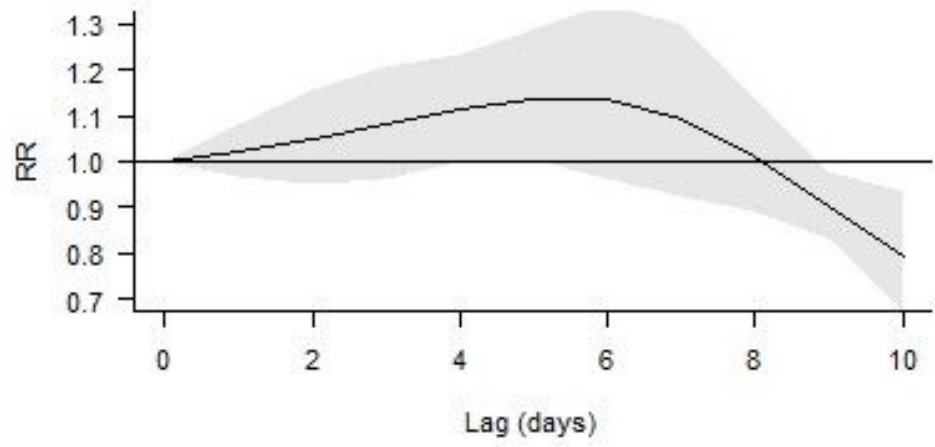

Temperature $=7^{\circ} \mathrm{C}$

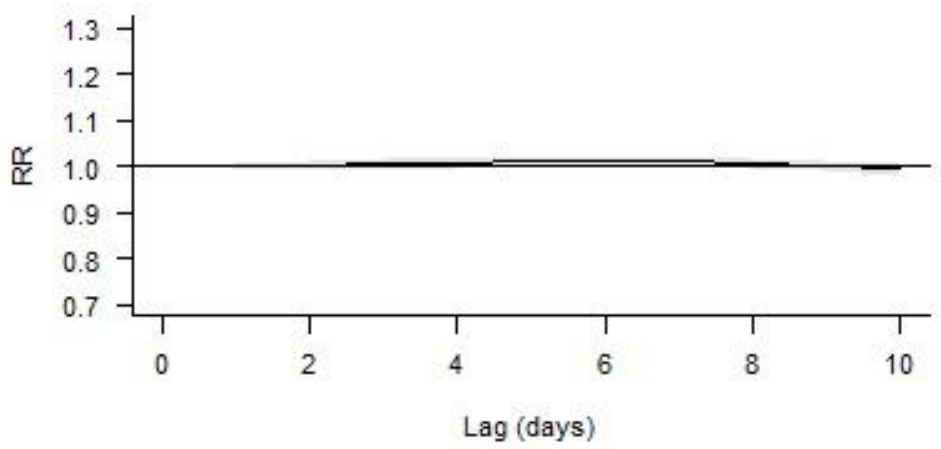

Temperature $=21^{\circ} \mathrm{C}$

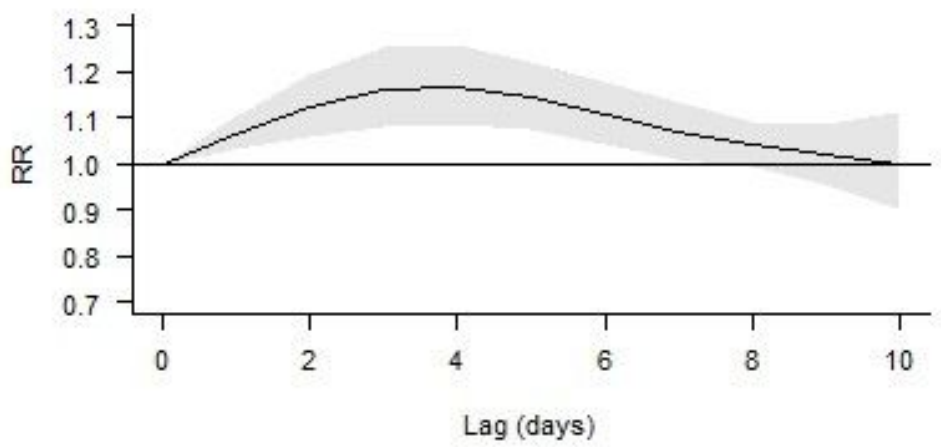

\section{Figure 3}

Exposure-specific effects of the lag-response association. The effects are centred at lag=0 and temperature $=4.7^{\circ} \mathrm{C}$. Grey shaded area represents $95 \%$ confidence intervals. 

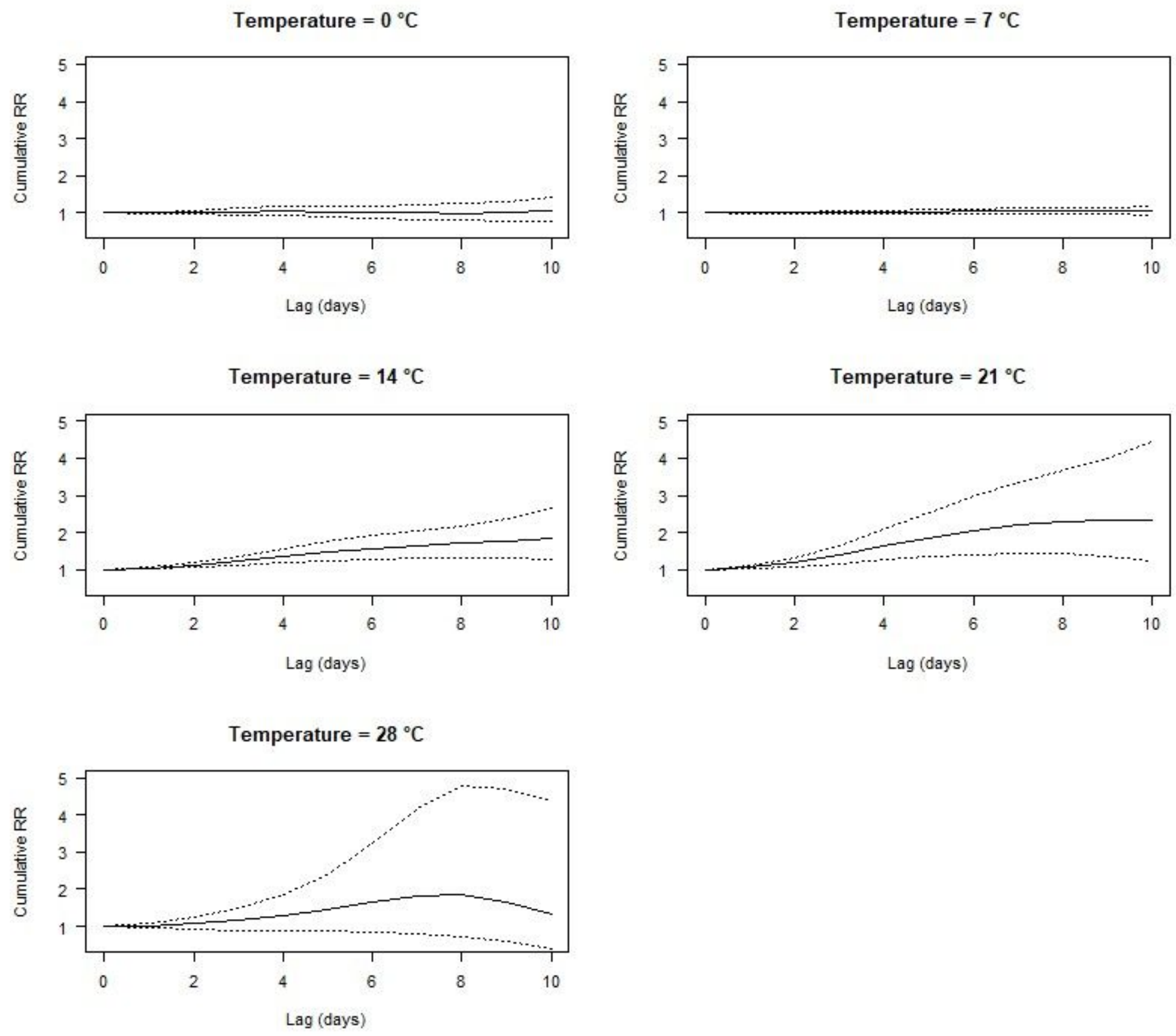

Figure 4

Overall exposure-specific lag-response association. The effects are centred at lag $=0$ days and temperature $=4.7^{\circ} \mathrm{C}$. Cumulative RR is computed by summing the log RR over the lag period ( 0 through 10 days inclusive). Dashed lines represent $95 \%$ confidence intervals.

\section{Supplementary Files}

This is a list of supplementary files associated with this preprint. Click to download.

- Supplementaryinformation.pdf 